\title{
Solution Structure of a Low-Molecular-Weight Protein Tyrosine Phosphatase from Bacillus subtilis
}

\author{
Huimin $\mathrm{Xu},{ }^{1,2}$ Bin Xia, ${ }^{1,2,3}$ and Changwen $\mathrm{Jin}^{1,2,3 *}$ \\ Beijing Nuclear Magnetic Resonance Center ${ }^{1}$ and College of Life Sciences ${ }^{2}$ and College of Chemistry and \\ Molecular Engineering, ${ }^{3}$ Peking University, Beijing 100871, China
}

Received 18 July 2005/Accepted 14 November 2005

\begin{abstract}
The low-molecular-weight (LMW) protein tyrosine phosphatases (PTPs) exist ubiquitously in prokaryotes and eukaryotes and play important roles in cellular processes. We report here the solution structure of YwlE, an LMW PTP identified from the gram-positive bacteria Bacillus subtilis. YwlE consists of a twisted central four-stranded parallel $\beta$-sheet with seven $\alpha$-helices packing on both sides. Similar to LMW PTPs from other organisms, the conformation of the YwlE active site is favorable for phosphotyrosine binding, indicating that it may share a common catalytic mechanism in the hydrolysis of phosphate on tyrosine residue in proteins. Though the overall structure resembles that of the eukaryotic LMW PTPs, significant differences were observed around the active site. Residue Asp115 is likely interacting with residue Arg13 through electrostatic interaction or hydrogen bond interaction to stabilize the conformation of the active cavity, which may be a unique character of bacterial LMW PTPs. Residues in the loop region from Phe40 to Thr48 forming a wall of the active cavity are more flexible than those in other regions. Ala41 and Gly45 are located near the active cavity and form a noncharged surface around it. These unique properties demonstrate that this loop may be involved in interaction with specific substrates. In addition, the results from spin relaxation experiments elucidate further insights into the mobility of the active site. The solution structure in combination with the backbone dynamics provides insights into the mechanism of substrate specificity of bacterial LMW PTPs.
\end{abstract}

Protein phosphorylation and dephosphorylation are involved in the regulation of many cellular processes, such as cell growth, differentiation, and metabolism $(12,23,35)$. The formation and hydrolysis of phosphate esters in proteins play key roles in the signal transduction through which the external environmental stimuli are converted to internal cellular response $(35,37)$. Protein tyrosine kinase (PTK) and protein tyrosine phosphatase (PTP) catalyze such reversible reactions. The cellular activities and cell growth are under the precise control of PTKs and PTPs. Defective or incorrect regulations in such systems result in many human diseases and thus they are ideal targets for drug design $(24,55)$.

PTPs belong to the protein phosphatase superfamily and catalyze the hydrolysis of phosphate ester on tyrosine residue in proteins. Based on their molecular masses and biological activities, PTPs can be divided into three families: the highmolecular-weight (HMW) PTP family, whose members contain a conserved catalytic domain with a molecular mass of around $30 \mathrm{kDa}$; the dual-specificity PTP family, which hydrolyzes phosphoserine or phosphothreonine as well as phosphotyrosine; and the low-molecular-weight (LMW) PTP family proteins, which are present in the cytoplasm with a molecular mass of around $18 \mathrm{kDa}(11,40,50,51)$. All these families of PTPs share very low sequence identities except the signature motif of the active site, $\mathrm{C}(\mathrm{X})_{5} \mathrm{R}(\mathrm{S} / \mathrm{T})$, at the phosphate-binding site. In PTP families, this motif is a relatively flexible loop and is called the protein tyrosine phosphate-binding loop, or Ploop (51).

\footnotetext{
* Corresponding author. Mailing address: Beijing Nuclear Magnetic Resonance Center, Peking University, Beijing 100871, China. Phone: 8610-6275-6004. Fax: 86-10-6275-3790. E-mail: changwen@pku.edu.cn.
}

It is well known that LMW PTPs down-regulate the plateletderived growth factor (PDGF)-stimulated cell proliferation by dephosphorylating the PDGF receptor in eukaryotes $(1,4)$. Phosphorylation on the tyrosine residue of LMW PTP itself in the process of PDGF signal transduction is involved in cytoskeleton rearrangement and cell adhesion $(3,5)$.

To date, many microbial LMW PTPs, such as from Acinetobacter johnsonii, Escherichia coli, Klebsiella pneumoniae, Mycobacterium tuberculosis, and Staphylococcus aureus, have been identified (19, 27, 39, 43, 46). Enzyme assays indicated that these LMW PTPs could specifically catalyze the hydrolysis of phosphotyrosine. In $K$. pneumoniae, the specificity was also confirmed by the observation that the LMW PTP could dephosphorylate an endogenous kinase that was autophosphorylated on tyrosine (39). The biological functions in cells are still poorly understood, though some functional roles have been proposed. The first identified yeast LMW PTP, Stp1, was found to induce a severe growth-defective phenotype and was considered to be involved in the regulation of cell growth (31). The LMW PTPs in prokaryotes were proposed to be involved in the production and transport of exopolysaccharides and capsular polysaccharides, which are important virulence factors of pathogenic microbe $(8,18,39)$. In several bacterial species, PTKs and LMW PTPs constitute a pair and PTK is the specific endogenous substrate of LMW PTP, such as Yco6/ Yor5 from K. pneumoniae, Wzc/Wzb from E. coli, and Ptk/Ptp from $A$. johnsonii $(19,39,46)$. The encoding genes of the PTK and LMW PTPs are located in the same cluster that consists of genes related to biosynthesis of exopolysaccharide $(8,45)$.

Biochemical and structural studies have demonstrated that the eukaryotic LMW PTPs share a similar catalytic mechanism, and that common steps are involved in the process of 


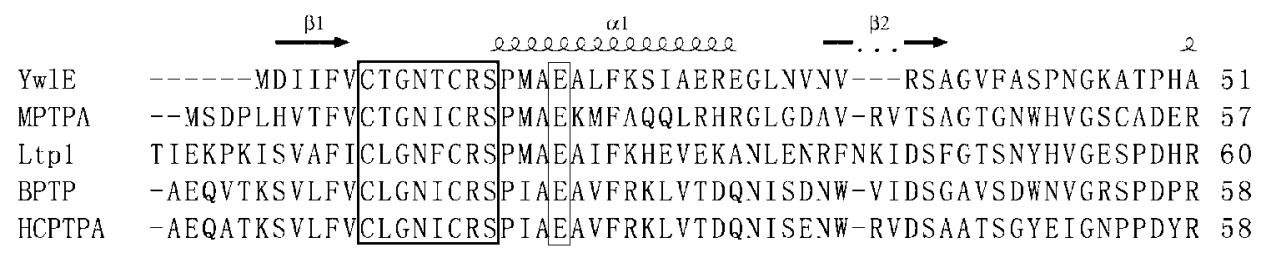

\begin{tabular}{|c|c|c|c|}
\hline & $\begin{array}{c}12 \\
\operatorname{eccepec}\end{array}$ & $282200222 \ldots$ e2 & \\
\hline 7 & VEALFE & & \\
\hline PTPA & MOVGTE & MLRS & \\
\hline $\operatorname{tp} 1$ & TVS & VCLFGD & 118 \\
\hline 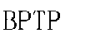 & JVTKEDF & LLGS & \\
\hline$P$ & ARQITKEDFA' & CMDESNLRDLNRKS & \\
\hline
\end{tabular}

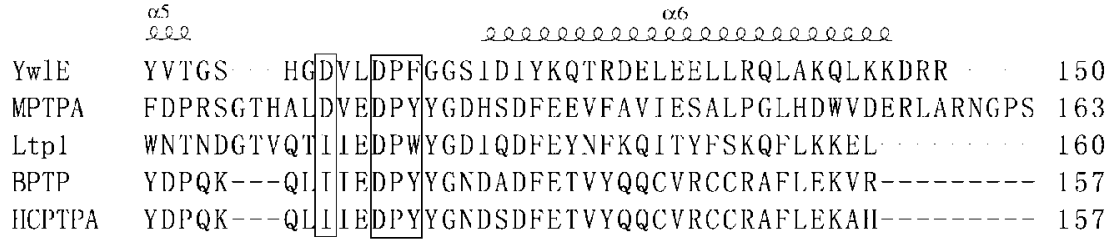

FIG. 1. Sequence alignment of LMW PTPs with B. subtilis YwlE, Mycobacterium tuberculosis MptpA (PDB code 1U2P), Saccharomyces cerevisiae Ltp1 (PDB code 1D1P), bovine PTP (BPTP; PDB code 1PNT), and human HCPTPA (PDB code 5PNT) by the program CLUSTAL W (22). The conserved residues in the P-loop and others mentioned in the text are highlighted in boxes. The protein sequences were obtained from the PDB database.

dephosphorylation (44, 48, 52). Bacterial LMW PTPs share low sequence identities with mammalian PTPs, whereas they exhibit high structural similarities.

The kinetics of the Bacillus subtilis PTK YwqD, the PTP YwqE, and the LMW PTPs YwlE and YfkJ were characterized $(32,33)$. The studies indicated that YwlE shows similar activity towards the substrate $p$-nitrophenylphosphate (pNPP), but a lower activity on the protein substrate of YwqE. A recent genetic and biochemical study suggested that YwlE plays roles in B. subtilis stress resistance (36). In order to explore the structural basis of bioactivity and further understand the binding mechanism of its specific endogenous substrate, we here report the solution structure and dynamic and kinetic properties of an LMW PTP, YwlE from B. subtilis. A detailed structural comparison with other LMW PTPs provides insights into the catalytic mechanism, especially the interactions with the specific substrates.

\section{MATERIALS AND METHODS}

Sample preparation. The $y w l E$ gene from B. subtillis was cloned into vector pET21a (Novagen). Uniformly ${ }^{15} \mathrm{~N} /{ }^{13} \mathrm{C}$-labeled and ${ }^{15} \mathrm{~N}$-labeled proteins were overexpressed in E. coli strain $\mathrm{BL} 21(\mathrm{DE} 3)$ at $37^{\circ} \mathrm{C}$ in $\mathrm{M} 9$ minimal medium containing ${ }^{15} \mathrm{~N}$-labeled ammonium chloride in the presence or absence of ${ }^{13} \mathrm{C}$ labeled glucose for ${ }^{15} \mathrm{~N}$-labeled and ${ }^{13} \mathrm{C}$-labeled or ${ }^{15} \mathrm{~N}$-labeled samples, respec-
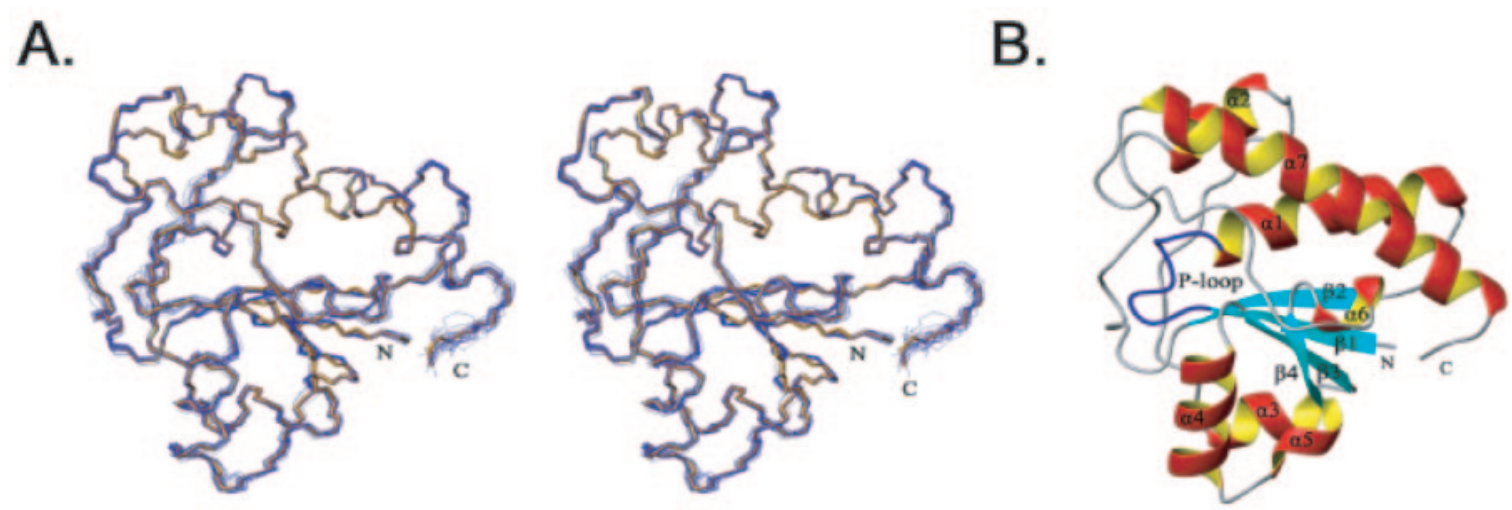

FIG. 2. Solution structure of B. subtilis YwlE. (A). The stereo view diagram of the superimposition of 20 representative structures (blue) with the mean structure (orange) of YwlE. (B). The ribbon diagram of the mean structure shows the secondary-structure elements. The $\mathrm{P}$-loop is in blue. 
tively. The soluble protein was purified by anion-exchange chromatography (Mono Q column) followed by gel filtration (Superdex-75 column) with the ÄKTA fast protein liquid chromatography (FPLC) system (Pharmacia). The protein purity is above $95 \%$ as judged by sodium dodecyl sulfate- polyacrylamide gel electrophoresis (PAGE). Nuclear magnetic resonance (NMR) samples were prepared in $50 \mathrm{mM}$ Tris- $\mathrm{HCl}$ buffer ( $\mathrm{pH} 7.5)$ and $50 \mathrm{mM}$ sodium phosphate buffer ( $\mathrm{pH} 7.5)$, respectively. Two-dimensional ${ }^{15} \mathrm{~N}$-edited heteronuclear single quantum coherence (HSQC) spectra showed identical numbers but different relative intensities of cross peaks in the absence and presence of phosphate. The NMR samples for structure determinations and dynamics were prepared in $50 \mathrm{mM}$ Tris- $\mathrm{HCl}$ buffer containing $50 \mathrm{mM}$ $\mathrm{NaCl}, 30 \mathrm{mM}$ dithiothreitol (DTT) ( $\mathrm{pH} 7.5$ ) in $90 \% \mathrm{H}_{2} \mathrm{O} / 10 \% \mathrm{D}_{2} \mathrm{O}$, and 2,2dimethyl-2-silapentane-5-sulfonic acid was added as the internal chemical shift reference. The NMR samples with a concentration about $1 \mathrm{mM}$ were used for structure determinations, while samples with different concentrations $(1.0,0.5,0.25$, and 0.1 $\mathrm{mM}$ ) were prepared for dynamics studies.

NMR spectroscopy. All NMR experiments were performed at $25^{\circ} \mathrm{C}$ on Bruker Avance $500-\mathrm{MHz}$ (with cryoprobe) and $800-\mathrm{MHz}$ spectrometers equipped with triple-resonance probes and pulsed-field gradients. The spectra were processed using software package NMRPipe (10) and analyzed using NMRView (25). Nearly complete backbone and side chain resonance assignments have been obtained and reported previously (49). Briefly, three-dimensional triple-resonance spectra HNCA, HN(CO)CA, HNCACB, CBCA(CO)NH, HBHA(CO)NH, $\mathrm{HNCO}$, and $\mathrm{HN}(\mathrm{CA}) \mathrm{CO}$ were collected to obtain the backbone resonance assignments. The three-dimensional spectra $\mathrm{H}(\mathrm{CC})(\mathrm{CO}) \mathrm{NH}$-total correlation spectroscopy (TOCSY), (H)CC(CO)NH-TOCSY, HCCH-TOCSY, CCH-TOCSY, HCCHcorrelation spectroscopy (COSY), CCH-COSY, and ${ }^{15} \mathrm{~N}$-edited TOCSY-HSQC were recorded for the side chain resonance assignments. The three-dimensional ${ }^{15} \mathrm{~N}$ and ${ }^{13} \mathrm{C}$-edited nuclear Overhauser effect spectroscopy (NOESY)-HSQC (mixing times of 100 and $50 \mathrm{~ms}$ ), experiments were performed to confirm the assignments and generate distance restraints for structure calculations. The hydrogen-deuterium (H-D) exchange experiments were performed to obtain the hydrogen-bonding information.

Structure calculations. The structure was determined using NOE-derived distance restraints, in combination with dihedral angle restraints and hydrogen bond restraints. The backbone dihedral angle restraints were determined using the program TALOS (7). Hydrogen bond distance restraints were generated from the H-D exchange experiments in conjunction with the intermediate-range NOEs and the secondary-structure information (41). The initial structures of YwlE were calculated using the module CANDID of the program CYANA by using NOE-derived distance restraints and dihedral angle restraints $(20,21)$. First, 50 structures were calculated and the 10 lowest-energy structures were selected as the filter models. The program SANE was employed to obtain further assignments semiautomatically from three-dimensional NOESY spectra based on the chemical shift assignments and predicted secondary-structure information (14).

The subsequently regenerated distance restraints by SANE were used as input for the structure calculations by the program CYANA. A total of 200 structures were calculated using CYANA. The 100 structures with the lowest target function values were selected and refined by AMBER7 using the force field ff99 and Generalized Born solvation model (38). The same restraints for the last run of CYANA were used. A 30-picosecond simulated annealing from 1,000 to $0 \mathrm{~K}$ was performed. The force constants for the NOE distance, dihedral angle, and hydrogen bond restraints were $20 \mathrm{kcal} \cdot \mathrm{mol}^{-1} \AA^{-2}, 50 \mathrm{kcal} \mathrm{mol}{ }^{-1} \cdot \mathrm{rad}^{-2}$ and $40 \mathrm{kcal} \cdot \mathrm{mol}^{-1} \AA^{-2}$, respectively. The chirality and transpeptide $\omega$ restraints with the force constants $150 \mathrm{kcal} \cdot \mathrm{mol}^{-1} \mathrm{rad}^{-2}$ and $50 \mathrm{kcal} \mathrm{mol}^{-1} \mathrm{rad}^{-2}$, respectively, were added to prevent chirality inversions or peptide bond flips during the high-temperature portions of simulated annealing (2).

During the structure calculations, several iterations of SANE-CYANA-AMBER were performed in conjunction with manual violation analysis. Finally, 20 structures with the lowest AMBER energies were selected to represent YwlE. The mean structure of the 20 structures was obtained by a 1,000-cycle energy minimization in vacuum using AMBER7. The program PROCHECK NMR was used to analyze the stereochemical geometry by Ramachandran statistics (28).

PTP activity assay. The phosphatase activity assays using pNPP as the substrate were performed in a total volume of $500 \mu \mathrm{l}$ containing $0.1 \mathrm{mM}$ sodium citrate (pH 6.5), $5 \mathrm{mM}$ DTT, $1 \mathrm{mM}$ EDTA, and $20 \mu \mathrm{g}$ YwlE at the optimized temperature of $55^{\circ} \mathrm{C}$. The initial rate of the reaction was measured by calculating the concentration of released $p$-nitrophenolate (pNP) ion using an absorbance at $405 \mathrm{~nm}$. The concentrations of pNPP used in the reaction ranged from 2 to 70 $\mathrm{mM}$. The total reaction time was $4 \mathrm{~min}$. The millimolar extinction coefficient was 18.

Titration experiments. The protein sample $(0.5 \mathrm{mM})$ was prepared in $50 \mathrm{mM}$ Tris- $\mathrm{HCl}$ buffer containing $50 \mathrm{mM} \mathrm{NaCl}$ and $30 \mathrm{mM}$ DTT at $\mathrm{pH}$ 7.5. Sodium phosphate was added at concentrations ranging from 1 to $50 \mathrm{mM}$. The phos-
TABLE 1. Structural statistics of B. subtilis YwlE

\begin{tabular}{cc} 
Parameter & Value \\
\hline
\end{tabular}

No. of NOE-derived proton-proton distance restraints

Intraresidue unambiguous $\mathrm{NOEs}^{a}$

Sequential unambiguous $\mathrm{NOEs}^{b}$

Medium-range unambiguous $\mathrm{NOEs}^{c}$

Long-range unambiguous NOEs ${ }^{d}$

Total unambiguous NOEs

Total ambiguous $\mathrm{NOEs}^{e}$

No. of H-bond distance restraints

692

913

3,094

1,653

48

No. of Dihedral angle restraints $(\phi$ and $\psi)$

162

No. of Restraint violations (20 structures)

NOE-derived distance violation $(>0.2 \AA)$

Torsion angle violation $\left(>5^{\circ}\right)$

Energy

CYANA target function value ${ }^{f}\left(\AA^{2}\right)$

Mean AMBER energy $\left(\mathrm{kcal} \mathrm{mol}^{-1}\right)$

NOE distance restraint violation energy $\left(\mathrm{kcal} \mathrm{mol}^{-1}\right.$ )

Torsion angle restraint violation energy $\left(\mathrm{kcal} \mathrm{mol}^{-1}\right.$ )

MSD from mean structure $(\AA)$

Backbone heavy atoms ${ }^{g}$

All residues

$0.38 \pm 0.07$

Secondary structures

$0.26 \pm 0.04$

All heavy atoms ${ }^{h}$

All residues

Secondary structures

$0.93 \pm 0.11$

$0.81 \pm 0.07$

Ramachandran statistics

Residues in most favored regions $(\%)$

Residues in additional allowed regions (\%)

Residues in generously allowed regions (\%)

87.2

11.2

Residues in disallowed regions (\%)

0.6

${ }^{a}$ Intraresidue NOEs represent the distance restraint between two protons in the same residue.

${ }^{b}$ Sequential represents the distance restraint between two protons that belong to sequential residues.

${ }^{c}$ Medium range represents two protons belonging to residues separated by no more than five amino acids.

${ }^{d}$ Long range represents protons belonging to residues separated by more than five amino acids in the primary sequence.

${ }^{e}$ Ambiguous NOEs represent NOE-derived distance restraints that could be assigned to more than one pair of protons.

$f$ The mean value of 20 structures with the lowest target function values out of 100 structures calculated with CYANA.

${ }^{g}$ The backbone heavy atoms include $\mathrm{C}^{\alpha}$, amide nitrogen atom, and carbonyl carbon atom.

${ }^{h}$ All heavy atoms include all nonhydrogen atoms in the protein.

phate-binding site was determined using the chemical shift changes from a series of two-dimensional ${ }^{15} \mathrm{~N}$-edited HSQC spectra at $25^{\circ} \mathrm{C}$. The composite chemical shift changes of amide ${ }^{15} \mathrm{~N}$ and ${ }^{1} \mathrm{H}$ were calculated using $\Delta \delta_{\text {comp }}=\left[\Delta \delta^{2}{ }_{\mathrm{HN}}+\right.$ $\left.\left(\Delta \delta_{\mathrm{N}} / 6\right)^{2}\right]^{1 / 2}$, where $\Delta \delta_{\mathrm{HN}}, \Delta \delta_{\mathrm{N}}$, and $\Delta \delta_{\text {comp }}$ are the ${ }^{1} \mathrm{H},{ }^{15} \mathrm{~N}$, and composite chemical shift changes of the cross peaks in the HSQC spectra, respectively (34).

Spin-relaxation measurements. The longitudinal and transverse relaxation rates $\mathrm{R}_{1}$ and $\mathrm{R}_{2}$, and steady-state heteronuclear $\left\{{ }^{1} \mathrm{H}\right\}-{ }^{15} \mathrm{~N}$ NOE values of YwlE were determined (16). The sample was in the same buffer conditions as that for structure calculations and four protein concentrations $(0.1,0.25,0.5$, and $1.0 \mathrm{mM})$ were used. The experiments were performed on a Bruker Avance 600-MHz NMR spectrometer at $25^{\circ} \mathrm{C}$. Spectral widths of $8,389.262 \mathrm{~Hz}$ for ${ }^{1} \mathrm{H}$ and $1,398.797 \mathrm{~Hz}$ for ${ }^{15} \mathrm{~N}$ were used and $512\left({ }^{1} \mathrm{H}\right)$ and $80\left({ }^{15} \mathrm{~N}\right)$ complex data points were collected for each experiment. Recycle delays of 2.2, 2.4, and 3.7 seconds were used for $\mathrm{R}_{1}, \mathrm{R}_{2}$, and NOE measurements, respectively. The delays used for $\mathrm{R}_{1}$ experiments were 100 (twice), 300 , $500,700,1000,1,500,2,000$, and 2,400 $\mathrm{ms}$, and $64,48,32$, and 16 transients per increment were used at sample concentrations of $0.1,0.25,0.5$, and $1.0 \mathrm{mM}$, respectively. The delays used for $\mathrm{R}_{2}$ experiments were 20 (twice), 40, 60, 80, 100, 120, 140, 


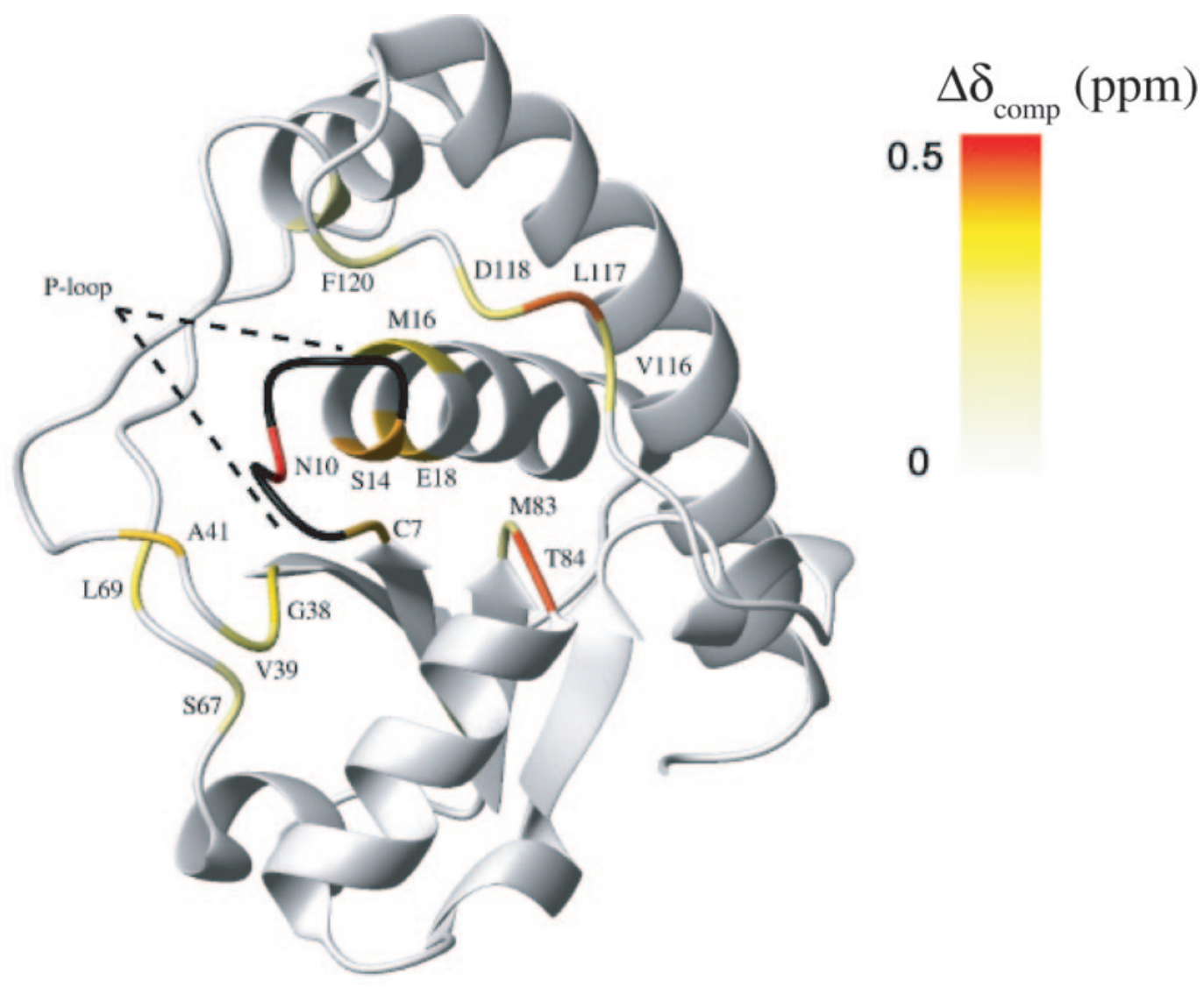

FIG. 3. Mapping the amide chemical shift changes on the ribbon diagram of YwlE. The color ranging from white to red corresponds to the composite chemical shift changes of amide ${ }^{1} \mathrm{H}$ and ${ }^{15} \mathrm{~N}$ from 0 to $0.5 \mathrm{ppm}$. The missing residues T8, G9, T11, C12, and R13 in the HSQC spectra are shown in black.

160 , and $180 \mathrm{~ms}$, and $64,64,16$, and 16 transients were used at the corresponding sample concentrations as for the $\mathrm{R}_{1}$ measurements. The relaxation rate constants were determined by fitting the peak intensities using the single exponential functions (17). The $\left\{{ }^{1} \mathrm{H}\right\}-{ }^{15} \mathrm{~N}$ NOE experiments were performed in the presence and absence of a proton presaturation period of 3 seconds prior to the ${ }^{15} \mathrm{~N}$ excitation pulse and using recycle delays of 3.7 and 6.7 seconds, respectively (16). The numbers of transient used were $128,96,64$, and 32 for the sample concentrations as in $\mathrm{R}_{1}$ and $\mathrm{R}_{2}$ experiments.

Protein structure accession numbers. The atomic coordinates of Bacillus subtilis YwlE have been deposited in the RCSB under PDB code 1ZGG. The chemical shift assignments have been deposited with BMRB accession number 6460 .

\section{RESULTS AND DISCUSSION}

Solution structure of YwlE. B. subtilis YwlE contains 150 amino acid residues and the sequence alignment of YwlE with other PTPs is shown in Fig. 1. The solution structure of YwlE has been determined using interproton NOE-derived distance restraints in combination with the dihedral angle restraints and hydrogen bond restraints. The 20 lowest-energy structures were selected to represent YwlE and are shown in Fig. 2A, together with the ribbon diagram showing the secondary-structure elements in Fig. 2B. The structural statistics are shown in Table 1. The root-mean-square deviation (RMSD) of backbone heavy atoms from the mean structure is $0.38 \pm 0.07 \AA$, $0.26 \pm 0.04 \AA$ for those in regular secondary structures. Only two NOE distance restraints show violation greater than 0.2 $\AA$ and no angle restraints show violation greater than $5^{\circ}$. For the 20 structures, $87.2 \%$ of the residues are in the most favored regions of the Ramachandran plot using PROCHECK_NMR (28), $11.2 \%$ are in the additionally allowed regions, $1.0 \%$ are in generously allowed regions, and $0.6 \%$ are in disallowed regions. The residue in the disallowed region is Asn10 in the P-loop, which is relatively flexible. Only few NOE-derived distance restraints were obtained for this residue.

YwlE comprises four $\beta$-strands (residues 2 to 6 [ $\beta 1$ ], 33 to 37 [ $\beta 2$ ], 79 to 82 [ $\beta 3$ ], and 102 to 104 [ $\beta 4]$ ), which form a twisted central parallel $\beta$-sheet, with six $\alpha$-helices (residues 14 to 28 [ $\alpha 1$ ], 51 to $57[\alpha 2], 71$ to $76[\alpha 3], 85$ to $95[\alpha 4], 107$ to $110[\alpha 6]$, and 124 to $145[\alpha 7]$ ) and a $3_{10}$ helix (residues 98 to $101[\alpha 5]$ ) flanking both sides. The highly conserved signature sequence from residues Cys7 to Ser14 forms the P-loop connecting $\beta 1$ and $\alpha 1$. Although YwlE shares a low sequence identity with LMW PTPs of known structures $(26 \%$ with human HCPTPA, $29 \%$ with bovine PTP [BPTP], 23\% with Saccharomyces cerevisiae Ltp1, and 31\% with Mycobacterium tuberculosis MptpA), the core packing of YwlE is highly similar to that of those PTPs. The RMSD values for the $\mathrm{C}^{\alpha}$ atom in the regular secondary-structure elements of YwlE from those of HCPTPA, BPTP, Ltp1, and MPtpA are 1.32, 1.26, 1.24, and $1.22 \AA$, respectively.

Mapping of the phosphate-binding site. The phosphate ion is a competitive inhibitor of LMW PTPs when pNPP is used as a substrate. To map the inhibitor-binding site, phosphate ion 
A.

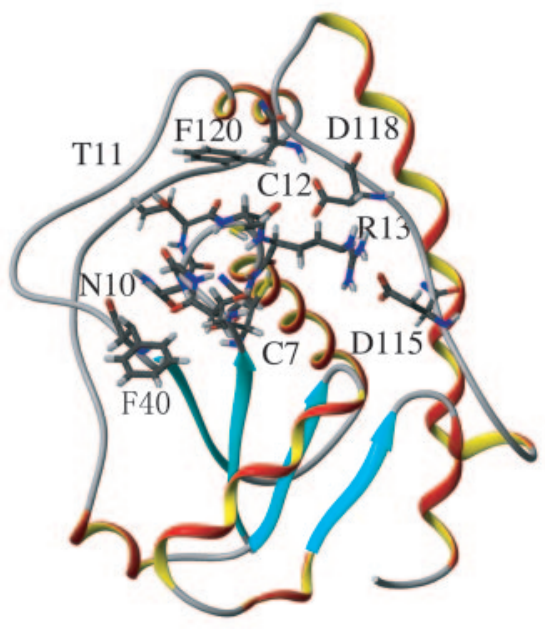

B.

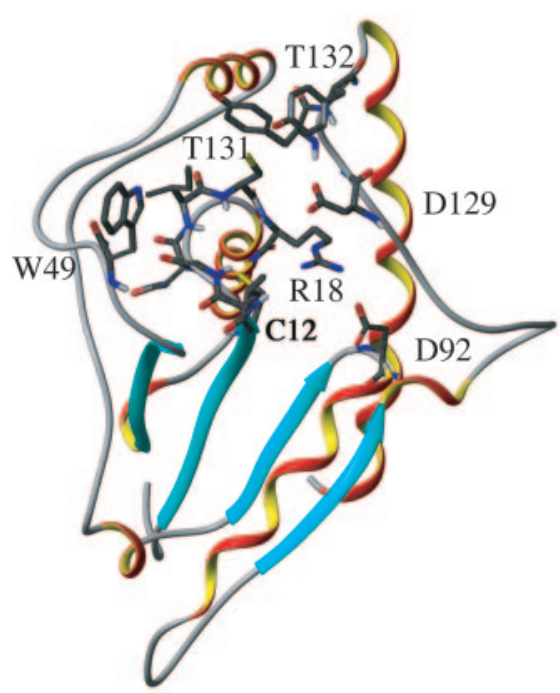

YwlE

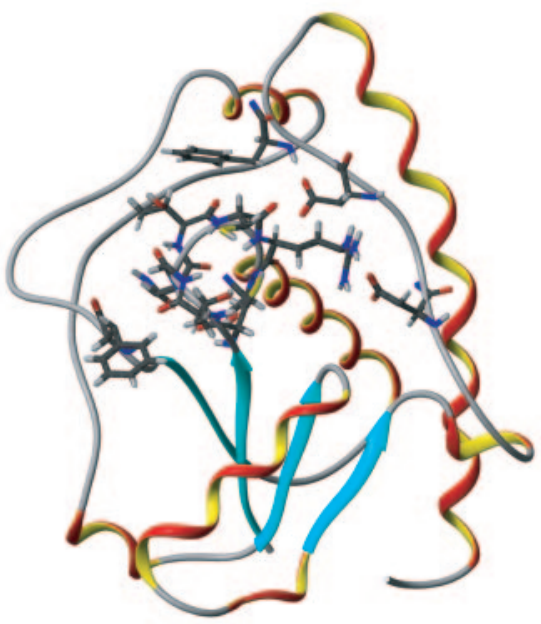

BPTP

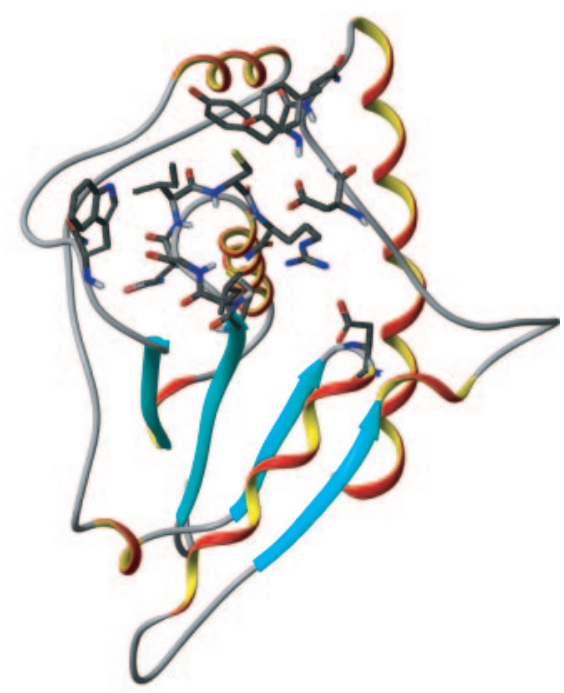

FIG. 4. Conformations of the active site. The active sites of YwlE and BPTP are shown in ball-and-stick style and the other regions of the proteins in the stereo view ribbon diagram. The N, H, C, O, and S atoms are shown in blue, gray, black, red, and yellow, respectively. (A). Active site of YwlE. It contains the P-loop (Cys7 to Ser14) and Asp118. Residues Phe40 and Phe120 are responsible for the selection of a tyrosine-containing substrate, and residue Asp115 stabilizes the local conformation of the active cavity by electrostatic interaction with Arg13, which are also shown. (B). Conformation of the active site of BPTP. Residues Cys12 and Arg18 in the P-loop region and Trp49, Asp92, Tyr131, and Tyr132 are shown.

titration experiments were performed and monitored by a series of two-dimensional ${ }^{1} \mathrm{H}_{-}{ }^{15} \mathrm{~N}$ HSQC spectra. Significant chemical shift changes were observed for residues Cys7, Asn10, Ser14, Met16, Glu18, Gly38, Val39, Ala41, Ser67, Leu69, Thr84, Val116, Leu117, Asp118, and Phe120. As shown in Fig. 3, these residues are located in the P-loop and neighboring regions surrounding the cavity formed by the P-loop. Among them, Asp118 is the putative proton donor, a key catalytic residue for the enzyme reaction. The results are in general agreement with similar observations on other LMW PTPs, indicating that the P-loop region of YwlE is the catalytic site $(29,48)$.
Active site. The active site of YwlE contains the P-loop and an aspartic acid residue, Asp118, that is nearby. The amino acid sequence of the P-loop in YwlE is CTGNTCRS, in which residues Cys7, Gly9, Asn10, Cys12, Arg13, and Ser14 are conserved in all LMW PTPs (Fig. 1). As shown in Fig. 4, most of the backbone N-H bonds in the P-loop point to the center of the phosphate-binding pocket, and Arg13 is located at the bottom of this pocket. This conformation suggests that strong hydrogen bond interactions might occur between the amide protons and the phosphate oxygen atoms of substrate.

The sequence alignments showed that Glu18 and His64 of 


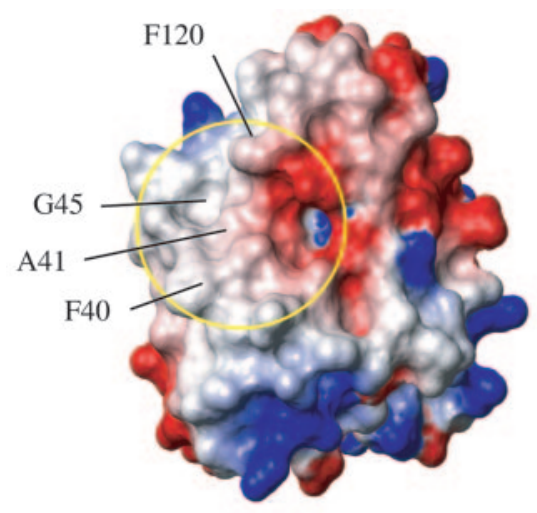

YwlE

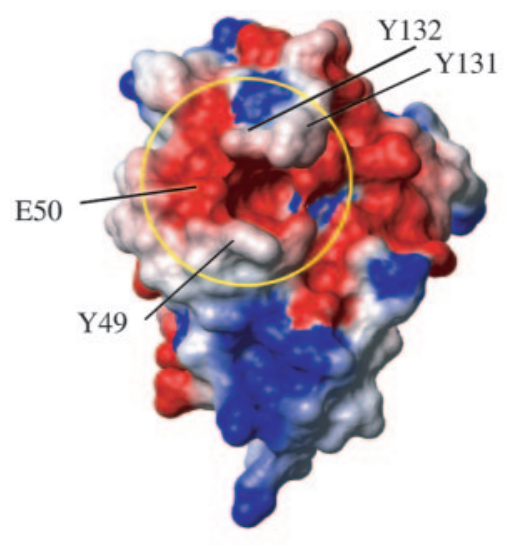

HCPTPA

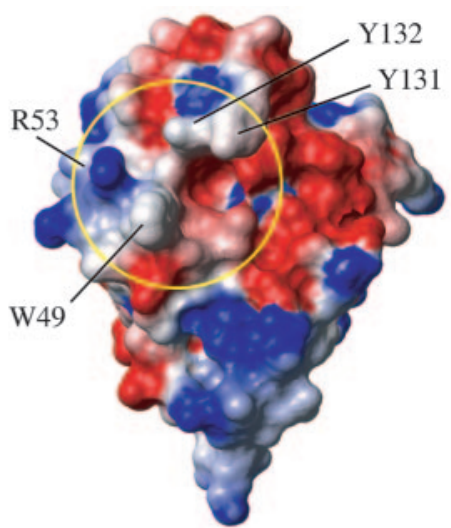

BPTP

FIG. 5. Charge distributions on the surface near the active sites of YwlE, HCPTPA, and BPTP. The positive charged regions are in blue, the negative charged regions are in red, and noncharged regions are in white. The active cavity and surface near it are highlighted in yellow circles.

YwlE were conserved among LMW PTPs with known structures (Fig. 1). Based on the structure of YwlE, Glu18 is close to the imidazole ring of His64 and the distance between $\mathrm{O}^{\varepsilon 1}$ of Glu18 and $\mathrm{N}^{\delta 1}$ of His64 is $3.71 \pm 0.68 \AA$, and it is $3.72 \pm 0.80$ $\AA$ between $\mathrm{O}^{\varepsilon 2}$ of Glu18 and $\mathrm{N}^{\delta 1}$ of His64. The spatial locations of Glu18 and His64 suggest that the electrostatic interaction or hydrogen bond interaction might occur between the two side chains and stabilize the conformation of the active cavity for binding with the phosphate group of the substrate. As shown in Fig. 4, the side chain hydroxide group of Asp118 is located at the top of the pocket, and the catalytic thiol of Cys7 is located at the bottom, which is geometrically similar to other LMW PTPs.

The structure of the active site provides insights into the enzyme activity, which was further confirmed by the enzyme activity assay. Previous studies demonstrated that both C7S and D118A mutations completely abolished enzyme activity (36). The highly conserved sequences and low RMSD values of $\mathrm{C}^{\alpha}$ atoms of YwlE and those of HCPTPA, BPTP, Ltp1, and MPtpA in the P-loop region $(0.79,0.83,0.86$, and $0.90 \AA$, respectively) further support that YwlE may share a similar catalytic mechanism with those LMW PTPs.

Residue Arg13 of YwlE in the P-loop is a signature of all LMW PTPs and plays an important role in enzyme activity. Previous studies indicated that the mutation R13K inactivated the enzyme completely (36). The corresponding residue in BPTP, Arg18, was proposed to be responsible for binding with the phosphate group and mutation of R18A abolished enzyme activity completely (9). Based on the structure of BPTP, the side chain atoms of Arg18 along with the backbone of the P-loop form a complete circle in which all amide $\mathrm{N}-\mathrm{H}$ bonds and $\mathrm{N}^{\varepsilon}-\mathrm{H}$ of $\operatorname{Arg} 18$ point to the center of the active cavity (53). The distances between $\mathrm{S}^{\gamma}$ of Cys12 and the amide nitrogen atoms of P-loop residues and $\mathrm{N}^{\varepsilon}$ of $\operatorname{Arg} 18$ are no more than 4.8 $\AA$. However, the positions of the side chain atoms of Arg13 in YwlE are relatively far from the sulfur atom of Cys7. The distance between $\mathrm{S}^{\gamma}$ of Cys7 and $\mathrm{N}^{\varepsilon}$ of $\operatorname{Arg} 13$ is $6.54 \pm 0.53 \AA$. No significant chemical shift perturbations of the side chain $\mathrm{N}^{\varepsilon}$ and $\mathrm{H}^{\varepsilon}$ of Arg13 were observed upon phosphate binding from the titration experiments. These results imply that Arg13 may not directly participate in the phosphate binding process in YwlE.

From the structure of YwlE, Asp115 is located near the guanidinium group of Arg13. The distance between $\mathrm{N}^{\eta^{2}}$ of $\operatorname{Arg} 13$ and $\mathrm{O}^{\delta 2}$ of Asp115 is $2.85 \pm 0.52 \AA$, and that between $\mathrm{N}^{\eta 1}$ of $\mathrm{Arg} 13$ and $\mathrm{O}^{\delta 1}$ of Asp115 is $2.76 \pm 0.50 \AA$. It is possible that the electrostatic or hydrogen bond interaction occurs between the guanidinium group of Arg13 and the carboxylate group of Asp115. The local conformation of the active cavity may be stabilized by the salt-bridge or hydrogen bond interaction between them. In all identified bacterial LMW PTPs, residue Asp115 is either conserved or replaced with a glutamate acid residue, whereas nonpolar residues are located at this position in mammalian ones. This fact suggests that the electrostatic or hydrogen bond interaction between Arg13 and Asp115 is likely a unique property of bacterial LMW PTPs. A similar electrostatic interaction in stabilizing the active pocket was found between Arg18 and Asp92 in BPTP (54). These results suggest that electrostatic and/or hydrogen bond interactions between the conserved Arg in P-loop and nearby Asp or Glu residues may play roles in the formation of the active site of LMW PTPs.

Substrate specificity. It is well known that substrate specificity is highly related to the local conformation of the active site and/or nearby regions. In LMW PTPs, loops between $\beta 2$ and $\alpha 2$ and that between the last two helices are considered to be involved in the recognition of and binding with phosphotyrosine-containing specific substrates $(30,52)$. The D-P-Y/ $\mathrm{W} / \mathrm{F}$ motif in the loop between the last two helices is also a conserved sequence in LMW PTPs. The hydrophobic nature and the orientation of the aromatic residue in this motif are important for the affinity of the enzyme towards different substrates. In YwlE, the aromatic ring of Phe120 extends the active cavity and forms a wall of the crevice for the incoming phosphotyrosine substrates. The loop between residues Phe40 and Thr48 forms another wall of the crevice (Fig. 5).

The positions of the hydrophobic and aromatic residues lining the walls of the active cavity suggest the enzyme may 
A.
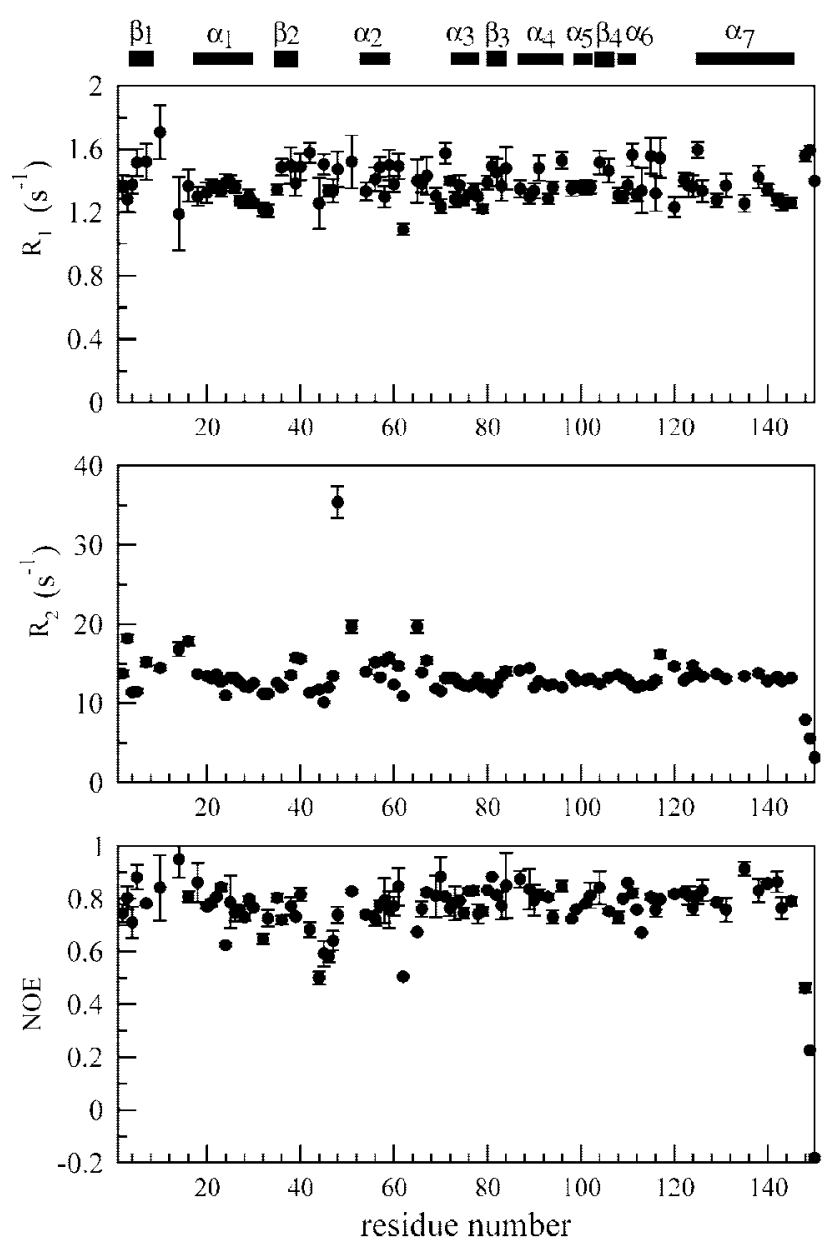

B.
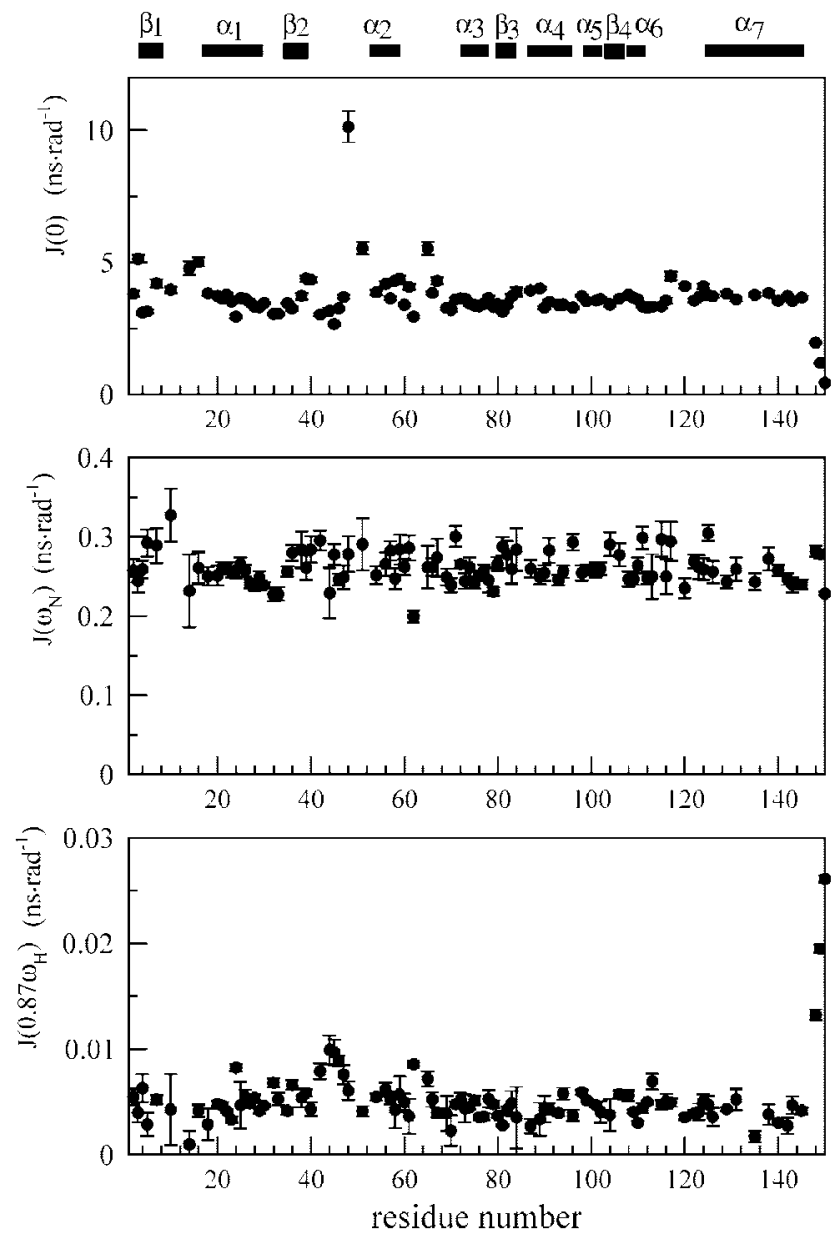

FIG. 6. Backbone dynamics of YwlE. (A) $\mathrm{R}_{1}, \mathrm{R}_{2}$, and $\left\{{ }^{1} \mathrm{H}\right\}-{ }^{15} \mathrm{~N}$ heteronuclear NOE and (B) reduced spectral density functions $J(0), J\left(\omega_{\mathrm{N}}\right)$, and $J\left(0.87 \omega_{\mathrm{H}}\right)$ values versus residue numbers. The regular secondary-structure elements are indicated on the top. The experiments were performed on a $600-\mathrm{MHz}$ NMR spectrometer at $25^{\circ} \mathrm{C}$. The sample $(0.25 \mathrm{mM})$ was dissolved in $50 \mathrm{mM}$ Tris- $\mathrm{HCl}$ buffer containing $50 \mathrm{mM} \mathrm{NaCl}$ and $30 \mathrm{mM}$ DTT at $\mathrm{pH} 7.5$.

specifically select the phosphotyrosine substrate instead of phosphoserine or phosphothreonine. Recent studies showed that YwlE was only active on phosphotyrosine-containing substrates when different substrates, including phosphotyrosine-, phosphoserine-, and phosphothreonine-containing peptides, were used, which is consistent with our proposal (36). In comparison with Trp49 in BPTP or Tyr48 in Ltp1, a nonaromatic residue, Ser42, occupies this position in YwlE. Though an aromatic residue, Phe 40, is closed to the active cavity, the phenylalanyl group of Phe 40 is too far away from that of Phe120 to stack for the hydrophobic interaction. The lower stacking interaction may be consistent with the high $K_{m}$ value of YwlE when pNPP is used as the substrate, which is several orders of magnitude higher than that of BPTP and Ltp1. Due to the hydrophilic residue Ser42, it is possible that sodium pyrophosphate can function as an inhibitor of YwlE, but not other LMW PTPs. Therefore, the concentration of pyrophosphate in B. subtilis may act as a regulator of YwlE activity.

The charge distribution around the active site is mainly contributed from the residues located in the loop between the $\beta 2$ and $\alpha 2$ (residues Phe40 to His50). Ala41 and Gly45 are closed to the active site and may be important for the selection of specific substrates. In eukaryotic LMW PTPs, residues closed to the active cavity are polar so that the surface near the active site is positive (BPTP) or negative (HCPTPA), whereas Ala41 and Gly45 in YwlE are nonpolar residues and contribute a noncharged surface around the active site (Fig. 5). Studies in mammalian LMW PTPs demonstrated that the charge distribution on the surface around the active site has a significant role in determining the specificities of the enzymes toward the substrates $(6,52)$. Thus, the presence of Ala41 and Gly45 indicates that YwlE might have natural peptides or protein substrates with fewer charges distributed on their surface.

Structural basis of the activity. The phosphatase activity was measured using pNPP, a nonphysiological phosphotyrosine analogue, as the substrate. The Michaelis-Menten constant $K_{m}$ is about $28 \mathrm{mM}$ and the rate constant $k_{\text {cat }}$ is about $0.14 \mathrm{~s}^{-1}$, which are much lower than that of eukaryotic LMW PTPs $(0.38 \mathrm{mM}$ for BPTP and $0.017 \mathrm{mM}$ for Ltp1). 
In the structure of YwlE, the position of the aromatic residue in other LWM PTPs that was lining the wall of the active cavity is replaced by serine, and in addition the side chain ring of Phe40 is not oriented favorably to form a closed crevice with Phe120. These structural differences may result in less stacking interaction between YwlE and pNPP. In addition, the conformation of the P-loop is different from that of BPTP and Ltp1. The N-H bond of Thr11 points away from the center of the pocket, which probably results in a smaller number of amide protons interacting with oxygen atoms of the phosphate group of the substrate, such as pNPP (Fig. 4). In comparison with Cys17 in BPTP and Cys18 in Ltp1, the side chain of Cys12 in YwlE flips into the active pocket, resulting in resistance in the space when the substrate interacts with the enzyme.

Internal dynamics. The longitudinal and transverse relaxation rates $\mathrm{R}_{1}$ and $\mathrm{R}_{2}$ and heteronuclear $\left\{{ }^{1} \mathrm{H}\right\}-{ }^{15} \mathrm{~N}$ NOE values were determined to investigate the motional properties of B. subtilis YwlE. The $\mathrm{R}_{1}, \mathrm{R}_{2}$, and $\mathrm{NOE}$ values versus the protein sequence are shown in Fig. 6A. We have calculated the $\tau_{c}$ values at sample concentrations of $0.1,0.25,0.5$, and $1.0 \mathrm{mM}$ with the program TENSOR2 (13) and the corresponding correlation time $\tau_{c}$ values were $8.83 \pm 0.16,9.37 \pm 0.13,9.65 \pm$ 0.15 , and $11.24 \pm 0.23 \mathrm{~ns}$, respectively, suggesting a weak oligomerization (dimmer) of YwlE at higher concentrations. In addition, YwlE showed a slightly axial anisotropy around 1.1, calculated using the structure and dynamics data.

The reduced spectral density function mapping approximation was used to analyze the experimentally determined relaxation data (15). In this approach, the calculated spectra density function $J(0), J\left(\omega_{\mathrm{N}}\right)$, and $J\left(0.87 \omega_{\mathrm{H}}\right)$ values can be used to analyze the overall and internal motions, where $J(0), J\left(\omega_{\mathrm{N}}\right)$, and $J\left(0.87 \omega_{\mathrm{H}}\right)$ represent the spectra density functions at frequencies of $0, \omega_{\mathrm{N}}$, and $\omega_{\mathrm{H}}$, respectively. The calculated $J(\omega)$ values for YwlE are shown in Fig. 6B. Since the values of $J(0)$ are normally dominated by that of $\mathrm{R}_{2}$, it is reasonable that the plots of $J(0)$ shows the trends of $\mathrm{R}_{2}$ versus residues. In addition, the $J(0)$ values are also contributed by the chemical exchanges $\mathrm{R}_{\mathrm{ex}}$.

The fairly constant values of $J\left(\omega_{\mathrm{N}}\right)$ and $J\left(0.87 \omega_{\mathrm{H}}\right)$ indicate that the protein is overall rigid except residues in the loop regions of Phe40-Ala51 and Leu62-Ser67 and the $\mathrm{C}$ terminus, where the residues show systematically higher $J\left(0.87 \omega_{\mathrm{H}}\right)$ values than those in other regions, indicative of a degree of flexibility on the picosecond to nanosecond timescales. Residues Thr48, Ala51, and Val65 and residues Ser14 and Met16 in the P-loop region show significantly large $J(0)$ values, suggesting conformational exchanges on a millisecond timescale. In addition, some residues in the P-loop were missing or unused due to the signal broadening and being undetectable or of poor quality for analysis, which arose from the intermediate conformational changes. Therefore, the $J(0)$ and $J\left(0.87 \omega_{\mathrm{H}}\right)$ values suggested that the residues in the $\mathrm{P}$-loop region and loop regions of Phe40-Ala51 and Leu62-Ser67 are involved in both subnanosecond internal motions and millisecond conformational exchanges (Fig. 6B). It is well known that enzyme activity is correlated with flexibility $(26,42,47)$. The flexibility of the $\mathrm{P}$-loop implies that it may influence the substrate-binding specificities.

Conclusions. The solution structure of YwlE shows the conserved structure and catalytic residues of the active site that catalyzes the hydrolysis of phosphate on tyrosine residue in its targets. Due to the conservations YwlE may function in a similar way in which the intermediate phosphocysteine enzyme is formed. The backbone structure of the P-loop is similar to that of mammalian LMW PTPs, whereas the side chain conformations are different. Unlike Arg18 in BPTP, Arg13 may not be directly involved in initial phosphate binding in YwlE. However, the sequence conservation and its possible stabilization by Asp115 suggest its importance in forming a phosphatebinding pocket.

The pair Arg13/Asp115 exists in all known bacterial LMW PTPs and is likely a hallmark of these enzymes. The flexibility of loops around the active site and noncharged surface resulting from Ala41 and Gly45 indicate that the hydrophobic interaction may occur between YwlE and the specific endogenous protein substrate. Recent studies on B. subtilis PTPs indicate that YwlE shows activity similar to that of YwqE towards pNPP but a lower activity on the substrate proteins of YwqE (33). However, YwlE is not located in the same operon as YwqE and the operon encoding YwlE remains largely unknown. Reduced resistance to ethanol stress resulting from genomic deletion of YwlE was observed and provided a clue in exploring the physiological roles of B. subtilis LMW PTPs (36). Further investigations are expected to characterize the specific endogenous protein substrates for YwlE and elucidate the role of Arg13 and Asp115.

\section{ACKNOWLEDGMENTS}

All NMR experiments were carried out at the Beijing Nuclear Magnetic Resonance Center (BNMRC), Peking University. We thank Xiao-Dong Su and Xiaofeng Zheng (Peking University) for help in the activity assays. We thank Ewen Lescop, Yunfei Hu, and Xianrong Guo (BNMRC) for discussions. We thank Xiubei Liao of the University of Illinois at Chicago for critical reading of the manuscript and suggestions.

This work was supported by the National Natural Science Foundation of China grant 30125009 (B.X.), grant 30325010 (C.J.), and grant 30570354 (C.J.).

\section{REFERENCES}

1. Berti, A., S. Rigacci, G. Raugei, D. Degl'Innocenti, and G. Ramponi. 1994 Inhibition of cellular response to platelet-derived growth factor by low M(r) phosphotyrosine protein phosphatase overexpression. FEBS Lett. 349:7-12.

2. Case, D. A., D. A. Pearlman, J. W. Caldwell, T. E. Cheatham III, J. Wang, W. S. Ross, C. L. Simmerling, T. A. Darden, K. M. Merz, R. V. Stanton, A. L. Cheng, J. J. Vincent, M. Crowley, V. Tsui, H. Gohlke, R. J. Radmer, Y. Duan, J. Pitera, I. Massova, G. L. Seibel, U. C. Singh, P. K. Weiner, and P. A. Kollman. 2002. AMBER 7, p. 130-131. University of California, San Francisco, Calif.

3. Chiarugi, P., M. L. Taddei, P. Cirri, D. Talini, F. Buricchi, G. Camici, G. Manao, G. Raugei, and G. Ramponi. 2000. Low molecular weight proteintyrosine phosphatase controls the rate and the strength of NIH-3T3 cells adhesion through its phosphorylation on tyrosine 131 or 132. J. Biol. Chem. 275:37619-37627.

4. Chiarugi, P., P. Cirri, G. Raugei, G. Camici, F. Dolfi, A. Berti, and G. Ramponi. 1995. PDGF receptor as a specific in vivo target for low $M_{r}$ phosphotyrosine protein phosphatase. FEBS Lett. 372:49-53.

5. Chiarugi, P., P. Cirri, M. L. Taddei, E. Giannoni, G. Camici, G. Manao, G. Raugei, and G. Ramponi. 2000. The low M(r) protein-tyrosine phosphatase is involved in Rho-mediated cytoskeleton rearrangement after integrin and platelet-derived growth factor stimulation. J. Biol. Chem. 275:4640-4646.

6. Cirri, P., T. Fiaschi, P. Chiarugi, G. Camici, G. Manao, G. Raugei, and G. Ramponi. 1996. The molecular basis of the differing kinetic behavior of the two low molecular mass phosphotyrosine protein phosphatase isoforms. J. Biol. Chem. 271:2604-2607.

7. Cornilescu, G., F. Delaglio, and A. Bax. 1999. Protein backbone angle restraints from searching a database for chemical shift and sequence homology. J. Biomol. NMR 13:289-302.

8. Cozzone, A. J., C. Grangeasse, P. Doublet, and B. Duclos. 2004. Protein phosphorylation on tyrosine in bacteria. Arch. Microbiol. 181:171-181. 
9. Davis, J. P., M. M. Zhou, and R. L. Van Etten. 1994. Kinetic and sitedirected mutagenesis studies of the cysteine residues of bovine low molecula weight phosphotyrosyl protein phosphatase. J. Biol. Chem. 269:8734-8740.

10. Delaglio, F., S. Grzesiek, G. W. Vuister, G. Zhu, J. Pfeifer, and A. Bax. 1995 NMRPipe: a multidimensional spectral processing system based on UNIX pipes. J. Biomol. NMR 6:277-293.

11. Denu, J. M., G. Zhou, Y. Guo, and J. E. Dixon. 1995. The catalytic role of aspartic acid-92 in a human dual-specific protein-tyrosine-phosphatase. Biochemistry 34:3396-3403.

12. DeVinney, R., O. Steele-Mortimer, and B. B. Finlay. 2000. Phosphatases and kinases delivered to the host cell by bacterial pathogens. Trends Microbiol. 8:29-33.

13. Dosset, P., J.-C. Hus, M. Blackledge, and D. Marion. 2000. Efficient analysis of macromolecular rotational diffusion from heteronuclear relaxation data. J. Biomol. NMR 16:23-28.

14. Duggan, B. M., G. B. Legge, H. J. Dyson, and P. E. Wright. 2001. SANE (Structure Assisted NOE Evaluation): an automated model-based approach for NOE assignment. J. Biomol. NMR 19:321-329.

15. Farrow, N. A., O. Zhang, A. Szabo, D. A. Torchia, and L. E. Kay. 1995 Spectral density function mapping using ${ }^{15} \mathrm{~N}$ relaxation data exclusively. J. Biomol. NMR 6:153-162.

16. Farrow, N. A., R. Muhandiram, A. U. Singer, S. M. Pascal, C. M. Kay, G Gish, S. E. Shoelson, T. Pawson, J. D. Forman-Kay, and L. E. Kay. 1994 Backbone dynamics of a free and a phosphopeptide-complexed Src homology 2 domain studied by ${ }^{15} \mathrm{~N}$ NMR relaxation. Biochemistry 33:5984-6003.

17. Fushman, D., S. Cahill, and D. Cowburn. 1997. The main-chain dynamics of the dynamin Pleckstrin homology (PH) domain in solution: Analysis of ${ }^{15} \mathrm{~N}$ relaxation with monomer/dimer equilibration. J. Mol. Biol. 266:173-194.

18. Grangeasse, C., B. Obadia, I. Mijakovic, J. Deutscher, A. J. Cozzone, and P. Doublet. 2003. Autophosphorylation of the Escherichia coli protein kinase Wzc regulates tyrosine phosphorylation of Ugd, a UDP-glucose dehydrogenase. J. Biol. Chem. 278:39323-39329.

19. Grangeasse, C., P. Doublet, C. Vincent, E. Vaganay, M. Riberty, B. Duclos, and A. J. Cozzone. 1998. Functional characterization of the low-molecular mass phosphotyrosine-protein phosphatase of Acinetobacter johnsonii. J. Mol Biol. 278:339-347.

20. Güntert, P., C. Mumenthaler, and K. Wüthrich. 1997. Torsion angle dynamics for NMR structure calculation with the new program DYANA. J. Mol. Biol. 273:283-298.

21. Herrmann, T., P. Güntert, and K. Wüthrich. 2002. Protein NMR structure determination with automated NOE assignment using the new software CANDID and the torsion angle dynamics algorithm DYANA. J. Mol. Biol. 319:209-227.

22. Higgins, D., J. Thompson, T. Gibson, J. D. Thompson, D. G. Higgins, and T. J. Gibson. 1994. CLUSTAL W: improving the sensitivity of progressive multiple sequence alignment through sequence weighting, position-specific gap penalties and weight matrix choice. Nucleic Acids Res. 22:4673-4680.

23. Hunter, T. 1995. Protein kinases and phosphatases: the yin and yang of protein phosphorylation and signaling. Cell 80:225-236.

24. Hunter, T. 2000. Signaling-2000 and beyond. Cell 100:113-127.

25. Johnson, B. A., and R. A. Blevins. 1994. NMRView: a computer program for the visualization and analysis of NMR data. J. Biomol. NMR 4:603-614.

26. Kay, L. E. 1998. Protein dynamics from NMR. Nat. Struct. Biol. 5(Suppl.): $513-517$.

27. Koul, A., A. Choidas, M. Treder, A. K. Tyagi, K. Drlica, Y. Singh, and A. Ullrich. 2000. Cloning and characterization of secretory tyrosine phosphatases of Mycobacterium tuberculosis. J. Bacteriol. 182:5425-5432.

28. Laskowski, R. A., J. A. C. Rullmann, M. W. MacArthur, R. Kaptein, and J. M. Thornton. 1996. AQUA and PROCHECK-NMR: programs for checking the quality of protein structures solved by NMR. J. Biomol. NMR 8:477-486.

29. Logan, T. M., M. M. Zhou, D. G. Nettesheim, R. P. Meadows, R. L. Van Etten, and S. W. Fesik. 1994. Solution structure of a low molecular weight protein tyrosine phosphatase. Biochemistry 33:11087-11096.

30. Madhurantakam, C., E. Rajakumara, P. A. Mazumdar, B. Saha, D. Mitra, H. G. Wiker, R. Sankaranarayanan, and A. K. Das. 2005. Crystal dtructure of low-molecular-weight protein tyrosine phosphatase from Mycobacterium tuberculosis at 1.9- $\AA$ resolutions. J. Bacteriol. 187:2175-2181.

31. Marchetta, M., T. Gamberi, S. Sarno, F. Magherini, G. Raugei, G. Camici, L. A. Pinna, and A. Modesti. 2004. Expression of the Stp1 LMW-PTP and inhibition of protein CK2 display a cooperative effect on immunophilin Fpr3 tyrosine phosphorylation and Saccharomyces cerevisiae growth. Cell. Mol. Life Sci. 61:1176-1184

32. Mijakovic, I., S. Poncet, G. Boël, A. Mazé, S. Gillet, E. Jamet, P. Decottignies, C. Grangeasse, P. Doublet, P. Le Maréchal, and J. Deutscher. 2003. Transmembrane modulator-dependent bacterial tyrosine kinase activates UDPglucose dehydrogenases. EMBO J. 22:4709-4718.
33. Mijakovic, I., L. Musumeci, L. Tautz, D. Petranovic, R. A. Edwards, P. R. Jensen, T. Mustelin, J. Deutscher, and N. Bottini. 2005. In vitro characterization of the Bacillus subtilis protein tyrosine phosphatase YwqE. J. Bacteriol. 187:3384-3390.

34. Mulder, F. A. A., D. Schipper, R. Bott, and R. Boelens. 1999. Altered flexibility in the substrate-binding site of related native and engineered high-alkaline Bacillus subtilisins. J. Mol. Biol. 292:111-123.

35. Mustelin, T., T. Vang, and N. Bottini. 2005. Protein tyrosine phosphatases and the immune response. Nat. Rev. Immunol. 5:43-57.

36. Musumeci, L., C. Bongiorni, L. Tautz, R. A. Edwards, A. Osterman, M. Perego, T. Mustelin, and N. Bottini. 2005. Low-molecular-weight protein tyrosine phosphatases of Bacillus subtilis. J. Bacteriol. 187:4945-4956.

37. Neel, B. G., and N. K. Tonks. 1997. Protein tyrosine phosphatases in signal transduction. Curr. Opin. Cell Biol. 9:193-204.

38. Pearlman, D. A., D. A. Case, J. W. Caldwell, W. S. Ross, T. E. Cheatham III, S. DeBolt, D. Ferguson, G. Seibel, and P. Kollman. 1995. AMBER, a package of computer programs for applying molecular mechanics, normal mode analysis, molecular dynamics and free energy calculations to simulate the structural and energetic properties of molecules. Comput. Phys. Commun. 91:1-41.

39. Preneta, R., S. Jarraud, C. Vincent, P. Doublet, B. Duclos, J. Etienne, and A. J. Cozzone. 2002. Isolation and characterization of a protein-tyrosine kinase and a phosphotyrosine-protein phosphatase from Klebsiella pneumoniae. Comp. Biochem. Physiol. B 131:103-112.

40. Ramponi, G., and M. Stefani. 1997. Structure and function of the low $\mathbf{M}_{r}$ phosphotyrosine protein phosphatases. Biochim. Biophys. Acta 1341:137156.

41. Reid, D. G., L. K. MacLachlan, A. J. Edwards, J. A. Hubbard, and P. J. Sweeney. 1997. Introduction to the NMR of proteins, p. 13-16. In D. G. Reid (ed.), Protein NMR techniques. Humana Press, Totowa, N.J.

42. Schnell, J. R., H. J. Dyson, and P. E. Wright. 2004. Structure, dynamics, and catalytic function of dihydrofolate reductase. Annu. Rev. Biophys. Biomol. Struct. 33:119-140.

43. Soulat, D., E. Vaganay, B. Duclos, A. L. Genestier, J. Etienne, and A. J. Cozzone. 2002. Staphylococcus aureus contains two low-molecular-mass phosphotyrosine protein phosphatases. J. Bacteriol. 184:5194-5199.

44. Su, X. D., N. Taddei, M. Stefani, G. Ramponi, and P. Nordlumd. 1994. The crystal structure of a low-molecular-weight phosphotyrosine protein phosphatase. Nature 370:575-578.

45. Vincent, C., B. Duclos, C. Grangeasse, E. Vaganay, M. Riberty, A. J. Cozzone, and P. Doublet. 2000. Relationship between exopolysaccharide production and protein-tyrosine phosphorylation in gram-negative bacteria. $\mathrm{J}$. Mol. Biol. 304:311-321.

46. Vincent, C., P. Doublet, C. Grangeasse, E. Vaganay, A. J. Cozzone, and B. Duclos. 1999. Cells of Escherichia coli contain a protein-tyrosine kinase, Wzc, and a phosphotyrosine-protein phosphatase, Wzb. J. Bacteriol. 181:34723477.

47. Volkman, B. F., D. Lipson, D. E. Wemmer, and D. Kern. 2001. Two-state allosteric behavior in a single-domain signaling protein. Science 291:24292433.

48. Wang, S., L. Tabernero, M. Zhang, E. Harms, R. L. Van Etten, and C. V. Stauffacher. 2000. Crystal structures of a low-molecular weight protein tyrosine phosphatase from Saccharomyces cerevisiae and its complex with the substrate $p$-nitrophenyl phosphate. Biochemistry 39:1903-1914.

49. Xu, H., P. Zhang, and C. Jin. 2005. NMR assignments of a low molecular weight protein tyrosine phosphatase (PTPase) from Bacillus subtilis. J. Biomol. NMR 31:363.

50. Yuvaniyama, J., J. M. Denu, J. E. Dixon, and M. A. Saper. 1996. Crystal structure of the dual specificity protein phosphatase VHR. Science 272: 1328-1331.

51. Zhang, M., C. V. Stauffacher, and R. L. Van Etten. 1995. The three dimensional structure, chemical mechanism and function of the low molecular weight protein tyrosine phosphatases, p. 1-23. In W. Merlevede (ed.), Advances in protein phosphatases, vol. 9. Leuven University Press, Leuven, Belgium.

52. Zhang, M., C. V. Stauffacher, D. Lin, and R. L. Van Etten. 1998. Crystal structure of a human low molecular weight phosphotyrosyl phosphatase. J. Biol. Chem. 273:21714-21720.

53. Zhang, M., R. L. Van Etten, and C. V. Stauffacher. 1994. Crystal structure of bovine heart phosphotyrosyl phosphatase at $2.2-\AA$ resolution. Biochemistry 33:11097-11105.

54. Zhang, Z., E. Harms, and R. L. Van Etten. 1994. Asp ${ }^{129}$ of low molecular weight protein tyrosine phosphatase is involved in leaving group protonation. J. Biol. Chem. 269:25947-25950.

55. Zhang, Z. Y. 2001. Protein tyrosine phosphatases: prospects for therapeutics. Curr. Opin. Chem. Biol. 5:416-423. 